\title{
Chapter 7 \\ Next Generation of CP: The Unity IT \\ Toolkit
}

\author{
Clara Ayora and Natasha Newton
}

\section{Introduction}

Traditionally, community policing (CP) constitutes an important tool for Law Enforcement Agencies (LEAs). Communities of people, despite their varying social, cultural, geographic, and ethnic differences, have common and shared values in their need for safety, security and wellbeing. Under the CP philosophy, LEAs are empowered to identify and solve problems proactively and jointly within their communities (Unity - Document of Work 2015).

However, despite the current technical connectivity, many citizens and their communities are disengaged from LEAs and other key stakeholders, which has a detrimental effect on CP. Coupling new technologies with traditional CP activities, for example foot patrol, leaflets, and local events, provides a means to further strengthen the cooperation between LEAs, Stakeholders, and Citizens. In this context, the Unity project was proposed. Its fundamental vision is to strengthen the connection between the LEAs and communities to maximize the safety and security of all citizens (Unity Deliverable 3.1 2015). This is achieved through a series of interlocking primary objectives:

- To capture best practices for cooperation between police and citizens;

- To design, develop and deliver training for LEAs and awareness raising activities about CP;

C. Ayora $(\bowtie)$

Treelogic. S.L., Madrid, Spain

e-mail: clara.aora@treelogic.com

N. Newton

Rinicom Ltd, Lancaster, UK

e-mail:natasha@ rinicom.com

(C) The Author(s) 2018 
- To develop a communications technology to facilitate, strengthen and accelerate the communication between citizens and police.

This chapter describes the technology used in Unity as a facilitator of CP against the 6 key pillars identified during the UNITY project; (1) trust and confidence building, (2) accountability, (3) information sharing and communication, (4) addressing local needs, (5) collaboration, and (6) crime prevention (Unity Deliverable 7.3 2016). The Unity IT Toolkit (herein 'ToolKit') is a suite of features that cover the best practices used to support and assist CP methods across all stakeholders (e.g., forums, news, events, instant messages, posting documents, social media and calendars, among others). These features work in tandem to provide an effective $\mathrm{CP}$ ToolKit. In addition, it is based on a number of modular design principles that support the flexibility, stability and robustness of the ToolKit, whilst ensuring security standards are complied with.

Given the variable casuistry of the different countries, the ToolKit needs to be instantiated for addressing each specific community (and therefore their specific needs and contexts). In particular, the ToolKit is parameterized and customized for eight live pilots within Croatia (month 6 of the project), Estonia (M11), Germany (M17), Belgium (M21), Finland (M26), Bulgaria (M29), Macedonia (M30) and the UK (M30). Their major goal is to test and validate with end users (i.e., LEAs, relevant stakeholders, and citizens) that $\mathrm{CP}$ could be enhanced by using the ToolKit, best practice processes and training. For each pilot, the ToolKit is adapted to the community's requirements adding or removing features as needed. In addition, to facilitate the mobility, the ToolKit is also implemented as a mobile app, taking advantage of the rich number of features that smart phones provide (e.g., bidirectional communication capabilities with the communities).

\section{Unity IT Toolkit Architecture}

The architectural design of the ToolKit ensures that a modular, flexible, extensible, scalable, robust and secure system is produced. The design process focused on facilitating the 6 pillars of CP using the technology as an enabler. This allowed for a trust-based, cooperative $\mathrm{CP}$ to be delivered in an efficient and effective way between LEA's, stakeholders and citizens. In addition, the bi-directional communication capabilities offered by mobile application technologies, combined with advanced data analytics enhances the LEA officers ability to make strategic decisions. The provision of 'other applications' (3rd party) has also been addressed and refers to integration with third party applications, this covers any application outside of the ToolKit which may interact by pushing/pulling information from it.

The architectural design places a strong emphasis on the user interface guidelines, which by default addresses the security analysis, by presenting a full threat model that uses the attacker's point of view to identify a list of threats that could undermine the trustworthiness of the system. 
The implementation of the Web Portal and Mobile Application (herein 'app') of the ToolKit takes into account the various views of the system, including; functional, data, business pro-cess, deployment, accessibility, usability, security, internationalisation/ localisation, resilience, performance and scalability. For the purpose of this chapter, focus will be placed on the functionality and usability of the web portal and app. The latter acts as a small screen wrapper of sorts, replicating the functions and features of the web portal, utilising the same functionalities, API calls and dependencies as its larger screened counterpart. Therefore, it is not necessary to duplicate the same functional view.

The Usability perspective addresses all aspects related to the User Interface of the ToolKit. The components that expose a User Interface include three main areas:

1. The header bar and footer areas - these areas are always displayed to the user on all pages. They include elements that may be of use to the user at any time. However, the actual contents in the header/ footer bars may change, depending on the user role.

2. The navigation menu - like the header bar, this area is always visible to the user on all pages. It allows the user to quickly navigate to the core pages of the portal, from anywhere in the portal. Again, as for the header bar, the options available in the navigation menu depend on the role of the user;

3. The contents area - this area is where the portal contents are presented. It is highly dynamic as it displays the contents of each web page. The user may vertically scroll through the displayed contents in this area.

The iOS and Android apps capture the primary functionalities of the portal itself, but with appropriate design, user interface and user experience considerations made to accommodate the unique functionalities, and in some cases limitations, of touch screen mobile devices.

The primary means of navigation within the app utilises a 'pull out' style map replicating the same basic layout of the web platform's navigation menu. As with the portal, the options provided to the user depends on their level of authentication within the system; anonymous, registered citizen, registered LEA/Stakeholder or Administrator. For the mobile implementation of the menu, a user can select from the pull-out list to open the precise area of the app they wish to view or use. The organisation of functionality and options within the app closely mirrors that of the web portal. The user can clearly see from the home-screen their respective levels of access within the app.

The use of cookies is a typical technique to improve the usability of the system for the users. However, in cases where this technique is used, when a user accesses the web portal for the first time a disclaimer is presented stating that the cookie policy is in use. If the user does not agree with the cookie policy, the cookies cannot be used. If the user agrees with the cookie policy, the disclaimer will not be displayed again. 
The contents accessible via the web portal depend on two aspects:

- The role of the user - different roles can access different functionality. This impacts the available options in the navigation bar, the information presented in the header and the web page contents;

- The selected community - users select their community of interest - usually a geographical local area but may be a virtual community too - and the web portal should only display contents that are relevant to the selected community.

The aim of the data driven analytics engine is to assist LEAs and other relevant stakeholders and stakeholders to explore and understand the issues and concerns underpinning the amount of information managed by Unity. Several technologies are involved to choreograph the functionality of the engine (e.g., MongoDB for the database, Python for implementing the features, Apache Tomcat for the web application). The engine supports the following features:

- A tag cloud: To identify topics, entities, organizations or locations in the ToolKit forum (e.g., what are people talking about in a thread). The importance of each tag is shown with different sizes and colours. The more relevant the term is, the bigger it appears;

- A sentiment analysis: To identify whether public opinion is evolving positively or negatively on a given subject

- A relationship graph: To identify cross relations among the tags of the tag cloud (e.g., two topics appear always together);

- External and public data mining: To identify similar interests between the users of the Toolkit and other external communities (e.g., Twitter or Facebook).

This engine is not conceived as an intelligence tool, instead it improves information sharing between LEAs and the communities in a transparent way as well as trust building by dealing with mid-term and long-term community-related problems.

Finally, the CP Strategy Generator (CPSG) is an intuitive tool developed with the purpose of achieving the following objectives:

- Accumulate and present all Unity research, analysis, and technology;

- Collect Current and Target operating information during each pilot and to provide a data repository for CP across Europe;

- Planning of CP strategy by LEA's

The CPSG is based loosely on strategic CP in the UK, encouraging users to look at their internal strategies in relation to Strategic, Tactical, and Operational improvements. The CPAG (Community Policing Architecture Glossary) framework is used to denote the potential capability areas users would look to improve with the CPSG, and from this, the Current and Target Operating Models are generated.

The CPSG takes on an iterative and inclusive approach, encouraging users to input any gaps in the data through their own experiences. The outcome is an autogenerated PDF report which users can review against their current strategies to determine any areas of improvement and enables data to be shared throughout Europe. 


\section{End User Assessment}

Given the variable casuistry of the different countries, the ToolKit is instantiated for addressing each specific community need and context. In particular, the ToolKit has been parameterized and customized for eight live pilots during the whole project. After each pilot, key focus groups were held with end users to understand perceived benefits after using an instantiation of the ToolKit.

The overall consensus was positive, end users advised they can see the real potential the ToolKit could have in real life scenarios, however due to the limited timeframes available for the pilots they were unable to test this theory effectively. In several cases direct comparisons to existing platforms were highlighted, including Facebook, Twitter, WhatsApp and Doodle poll. Unity is working towards integrating with exiting platforms where possible as it understands the potential barrier to adopting new technology, especially in older generations. That being said, with prior training and user manuals the user experience of the ToolKit could also be enhanced through a shared understanding of the benefits Unity provides.

As with all technology, there was a concern across all pilots regarding the possibility of end users misusing the ToolKit for criminal behaviour or as another way to report emergencies. All efforts have been taken to mitigate this risk by including disclaimers clearly highlighting the tool does not replace existing emergency channels, and that all communication will be monitored by LEA's.

Future pilots will seek input from citizens to review the marketability and trust within a community to use the proposed ToolKit. This places emphasis on the proper exploitation of the tool, ensuring end users buy-in to the concept and understand the real value of such a system. To achieve this, the LEA's within the consortium will liaise closely with their respective communities, providing them with the necessary training and support required.

\section{Conclusions}

The Unity IT ToolKit has been defined in the context of the Unity project that is intended to strengthen the connection between the LEAs and their communities to maximize the safety and security of all citizens. Through different views and perspectives, a set of features are implemented including forums, news, events, instant messages, posting documents, social media and calendars, among others. In addition, a data driven analytics engine has been included to support decisionmaking for all the different stakeholders to cooperatively plan actuations for improvement. Finally, the CP Strategy Generator is an intuitive tool to collect operating information, to provide a data repository for $\mathrm{CP}$, and to plan $\mathrm{CP}$ strategy by LEA's.

To facilitate its adoption, the ToolKit has been implemented as a desktop application and as a mobile application, both in iOS and Android. All the implemented 
features are parameterized and customized for eight live pilots, for which the major goal is to test and validate with end users that CP could be enhanced by using the ToolKit, best practice processes and training.

During key focus groups, end users envisioned the real potential of the ToolKit, identifying particular scenarios it would be beneficial. However, due to the limited timeframes available for trialling the ToolKit, users were unable to test this theory effectively. Also, they directly compare it to existing platforms such as WhatsApp. We consider that with prior training and user manuals, the user experience could be enhanced through a shared understanding of the Unity's benefits.

Going forwards, during the project, future instantiations of the ToolKit will be reevaluated by end users to gather a further understanding of the impact it could have on CP from a marketability and trust perspective. A detailed exploitation plan will be drafted as prat of the project, clearly identifying the route to commercialisation and it is expected the end user partners within the consortium will be the initial early adopters of the ToolKit post-project.

\section{References}

Unity - Document of Work. (2015). Grant Agreement No: 653729 - Horizon 2020. European Union.

Unity Deliverable 3.1. (2015). Report on existing approaches and best/effective practices to community policing. European Union Horizon 2020 Programme under grant agreement no 653729.

Unity Deliverable 7.3. (2016). Report on results of the baseline measurements. European Union Horizon 2020 Programme under grant agreement no 653729.

Open Access This chapter is licensed under the terms of the Creative Commons Attribution 4.0 International License (http://creativecommons.org/licenses/by/4.0/), which permits use, sharing, adaptation, distribution and reproduction in any medium or format, as long as you give appropriate credit to the original author(s) and the source, provide a link to the Creative Commons license and indicate if changes were made.

The images or other third party material in this chapter are included in the chapter's Creative Commons license, unless indicated otherwise in a credit line to the material. If material is not included in the chapter's Creative Commons license and your intended use is not permitted by statutory regulation or exceeds the permitted use, you will need to obtain permission directly from the copyright holder.

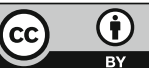

